\title{
A 2D Image-Based Approach for CFD Validation of Liquid Mixing in a Free-Surface Condition
}

\author{
P. E. Rodríguez-Ocampo ${ }^{1 \dagger}$, M. Ring ${ }^{1}$, J. V. Hernández-Fontes ${ }^{1}$, J. C. Alcérreca-Huerta ${ }^{2}$, \\ E. Mendoza ${ }^{1}$, G. Gallegos-Diez-Barroso ${ }^{3}$ and R. Silva ${ }^{1}$ \\ ${ }^{1}$ Institute of Engineering, National Autonomous University of Mexico (II-UNAM), Mexico City,04510, Mexico \\ ${ }^{2}$ Department of Systematics and Aquatic Ecology, National Council of Science and Technology-The Southern \\ Border College (CONACYT-ECOSUR), Chetumal, 77014, Mexico \\ ${ }^{3}$ Coastal Processes and Physical Oceanography Laboratory, Department of Marine Resources, Center for \\ Research and Advanced Studies of the National Polytechnic Institute (CINVESTAV), Merida, 97310, Mexico
}

†Corresponding Author Email: elipao29@hotmail.com

(Received August 28, 2019; accepted February 8, 2020)

\begin{abstract}
This study proposes an image-based approach to evaluate the validity of numerical results for cases where the setup can be assumed to be two-dimensional (2D) and mixing between liquids of different densities occurs under a free-surface condition. The proposed methodology is based on the estimation of the relative errors of the model through density matrices generated from images of the experimental and numerical results (i.e., postprocessing snapshots of the density field). To demonstrate the use of the methodology, experimental tests and numerical simulations were performed for a double-dam-break problem with two miscible liquids. For the experiments, a high-speed camera was employed to capture details of the fluid interactions after the dam breaking. For the numerical simulations, an OpenFOAM® multiphase solver was employed to reproduce the benchmarking tests. Three turbulence approaches were tested: a zero-equation RANS model, a two-equation (k-epsilon) RANS model, and a Large-Eddy Simulation (LES) model. The experimental results compared favorably against the numerical results, with averaged relative errors of $\sim 17$ and $\sim 19 \%$ for the zero-equation and the two-equation turbulence models, respectively, and $\sim 14 \%$ for the LES model. From the results obtained, it can be inferred that the two-equation (k-epsilon) model had limitations in reproducing the mixing between the liquid phases in terms of relative errors. The LES model reproduces the mixing between phases more accurately than zero and two-equation RANS models, which were seen to be more suitable for capturing the formation of large eddies in the initial phase of the experiment. The present methodology can be improved and extended for different multiphase flow configurations.
\end{abstract}

Keywords: Numerical modelling; Multiphase flow; Liquid-liquid-gas interface flows; Turbulence models; Validation; Dam-break experiments.

\section{NOMENCLATURE}

$\begin{array}{ll}\text { CC } & \text { central compartment } \\ \text { CFD } & \text { Computational Fluid Dynamics } \\ \text { CL } & \text { Left Compartment } \\ \text { CR } & \text { Right Compartment } \\ \text { D } & \text { diffusivity } \\ \mathrm{e} & \text { error per pixel } \\ \mathrm{g} & \text { acceleration due to gravity } \\ \mathrm{I} & \text { Kronecker delta } \\ \mathrm{k} & \text { turbulent kinetic energy per unit mass } \\ \mathrm{k} & \text { turbulent kinetic energy } \\ \text { LES } & \text { Large Eddy Simulation } \\ \mathrm{p} & \text { pressure } \\ \text { RANS } & \text { Reynolds Averaged Navier-Stokes } \\ & \text { Simulation } \\ \mathrm{S} & \text { strain rate tensor }\end{array}$

$\begin{array}{ll}\mathrm{S} 1 & \text { first part of the experiment } \\ \mathrm{S} 2 & \text { second part of the experiment } \\ t & \text { time } \\ \mathrm{U} & \text { flow velocity } \\ \mathrm{U}_{\mathrm{r}} & \text { relative velocity } \\ \mathrm{VOF} & \text { Volume of Fluid } \\ & \\ \alpha & \text { fluid phase } \\ \Delta \rho & \text { density gradient } \\ \varepsilon & \text { turbulent dissipation } \\ \mu & \text { dynamic viscosity } \\ \mu_{\mathrm{t}} & \text { dynamic eddy viscosity } \\ \rho & \text { density } \\ \rho_{w} & \text { freshwater density } \\ \rho_{s w} & \text { saltwater density }\end{array}$




$\begin{array}{ll}\overline{\rho_{\exp }} & \begin{array}{l}\text { averaged density matrix from the } \\ \text { laboratory tests } \\ \text { density matrix from the numerical } \\ \text { simulation }\end{array} \\ \rho_{\text {sim }} & \end{array}$

\section{INTRODUCTION}

The study of multiphase flows is important in several engineering areas such as mechanical, nuclear, civil, ocean, petroleum, environmental, and aerospace engineering. Nowadays, it is common to investigate multiphase flows through numerical modelling, for which experimental validations are required. Some examples of multiphase flow analyses in real applications can be found in the fields of medicine (e.g., the flow of blood in our body: Buradi and Mahalingam, 2016; Melka et al. 2018; Ostrowski et al. 2016; Sia et al. 2018); food (e.g., fermentation processes: Pessoa et al. 2016; Schmidt \& Velten, 2015, 2016); environment (e.g., gas emissions: Chang et al. 2013; Lamas et al. 2017; Moraiti et al. 2016); petroleum (e.g., multiphase flow through pipes: Ersoy et al. 2017; Leporini et al. 2018; Shi et al. 2018; Sun et al. 2016), turbomachinery (e.g., multiphase flow in turbines: Jiang et al. 2019; Lin et al. 2018; and multiphase flow in pumps: Bai et al. 2019a; Bai et al. 2019b), as well as thermal applications (e.g., heat transfer processes: Afrasiabi et al. 2018; Buliński et al. 2018; Cao et al. 2014; Kumar et al. 2017).

Since multiphase flow types have differences in pressures, temperatures, densities, and flow regimes, they require different configurations to perform validation and analyze the phenomena of interest. Thus, there is no specific experimental setup for the validation of all types of multiphase flow conditions. It would be complex to propose experimental facilities and methodologies to validate all multiphase flow configurations. Furthermore, there is still a lack of experimental facilities and procedures to analyze validations in some areas of multiphase flow research (Bello et al. 2007).

A typical means of contributing to the validation processes is to perform research into complex problems through simplified experimental configurations, which allow relevant data to be extracted for comparing numerical and experimental results. Examples of this type of simplification include the study of complex turbulent flows on decks of marine structures during harsh environmental conditions (e.g., Greco et al. 2007; Greco et al. 2005; Hernández-Fontes et al. 2018), effects of inundations inland due to tsunamis (e.g., Foster et al. 2017), or the sudden release of water reservoirs (e.g., Lobovský et al. 2014).

Computational Fluid Dynamics (CFD) methods are some of the most common tools used to study multiphase flows. These methods use computergenerated numerical solutions of the hydrodynamic governing equations. Then, to guarantee that CFD solutions agree with the real physics of the problems, validation is required (Eça \& Hoekstra, 2008; Eça et al. 2016; ITTC, 2008; Stern et al. 2006; Stern et al.

$\begin{array}{ll}\sigma & \text { surface tension } \\ \tau & \text { specific Reynolds stress tensor } \\ \Phi & \text { scalar quantity }\end{array}$

2001)

USDD (Department of Defense, 2009) defines "validation" as the process of determining the degree to which a model or simulation and its associated data are an accurate representation of the real world from the perspective of the intended uses of the model. To the best of the authors' knowledge, approaches to evaluate the validity of CFD simulations for stages where mixing between phases occurs are scarcer.

The present paper aims to offer an image-based approach for comparing numerical and experimental results through the analysis of the relative errors produced; thus, contributing to a preliminary validation of the numerical simulation. Based on results by Rodríguez-Ocampo (2016), the 2D image approach focuses on liquid-liquid-gas interface flows, which can be relevant in hydrodynamic applications by considering miscible fluids of different densities and a free-surface condition. The study case consists of a double-dam-break problem, assumed to be two-dimensional, where fluids of different density and hydraulic head interact following the sudden release of two dams. Systematic experimental runs were performed under a controlled environment to ensure the repeatability of the tests. Fluid interaction details were captured with a high-speed camera. To reproduce numerically the benchmarking experimental case, the opensource OpenFOAM® software was employed, considering three different turbulence models.

The main steps of the methodology are presented in Section 2, whereas Sections 3 and 4 describe the details of the experimental and numerical methods, respectively. The results are shown in Section 5. Section 6 corresponds to discussion and recommendations to improve and extend the method. Finally, the conclusions are drawn in Section 7.

\section{Proposed Methodology}

The main stages of the proposed image-based multiphase CFD validation method are described in this section. Fig. 1 presents the workflow of the proposed method, which is defined by eight steps. It starts with the image data acquisition from experimental and numerical results as the central basis for the generation of error matrices and the analysis of differences between numerical and experimental results.

The steps in Fig. 1 are detailed as follows:

i. The first step consists in acquiring image-based results from both experiments and CFD simulations. For this purpose, a representative experiment is to be designed and constructed to include the variables to be studied (e.g., different 
phases, mixing, free-surface). The study case is set and repeated several times to reduce experimental errors and the random component of each experiment. The experiments are recorded by a high-speed camera. The different fluid phases are differentiated by coloring them, but, for the sake of simplicity, gray-scale images could be used. The illumination of the experiment must be carefully set to avoid shadows that affect the image processing. The experiments that were incorrectly performed are discarded. On the other hand, the experimental setup is to be reproduced numerically with a CFD software to obtain post-processed images of the density field.

ii. Image edition: The experimental data (images) captured by the camera and the simulation results are cropped to keep the area of interest only, providing focus on the fluid motion.

iii. Frequency matching: If required, subsampling must be done to match the time steps between the experiment and the simulation. In this way, the number of simulation snapshots will correspond with the recorded frames of the experiment.

iv. Pixel matching: If necessary, to obtain the same amount of pixels, interpolation should be performed between the resolution of the images from numerical results and images from experiments.

v. Calibration at time-zero: Each of the images obtained experimentally are calibrated according to the time-zero frame, i.e., the first frame before the start of the experiment. For this purpose, a density value $\rho_{1}$ is given to a pixel/region color within the region with phase 1 , while a density $\rho_{2}$ is assigned to a pixel/region color of a section with phase 2 . The color scale is thus calibrated based on a linear interpolation between $\rho_{1}$ and $\rho_{2}$.

vi. Generation of density matrices: A density value, according to the calibration, is given to each pixel of the images obtained in the previous steps. This process is repeated for each time step.

vii. Average of the experimental density matrices (optional): This step consists in averaging the density matrices of all the experiments performed and at each time step. The process can be performed if the experiment was repeated several times, and if it is pertinent to have one single averaged experimental result.

viii. Generation of error matrices: The averaged density matrix from the laboratory tests $\left(\overline{\rho_{\exp }}\right)$ is compared against the density matrix from the numerical simulation $\left(\rho_{\text {sim }}\right)$ for each time step. The absolute error is then obtained by considering the absolute value of the difference between $\overline{\rho_{\text {exp }}}$ and $\rho_{\text {sim }}$ for every pixel, per frame. This difference is then divided by the density gradient between liquid phases $(\Delta \rho)$ to obtain the relative error, as shown in Eq. (1). If needed, the error per pixel considering Eq. (1) can be plotted with a color scale.

$$
\mathrm{e}=\left|\frac{\overline{\rho_{\text {exp }}}-\rho_{\text {sim }}}{\Delta \rho}\right| \times 100
$$

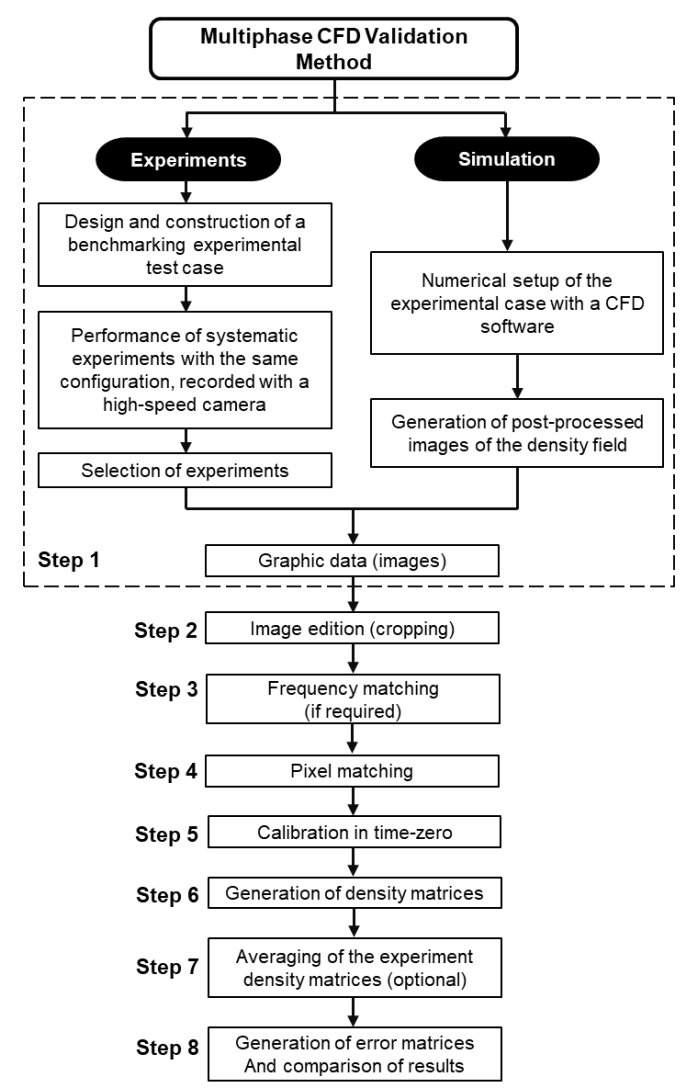

Fig. 1. Stages of the proposed image-processing multiphase CFD validation method.

It is possible to estimate averaged relative errors in the complete domain where the fluid interactions occur, in particular regions of the domain, or at specific points.

Furthermore, the free-surface can be tracked through each time step in the density matrices, providing an indirect method for free-surface tracking. This process is based on obtaining a binary matrix, where the value of 1 (or "true") is given to the area filled with liquid and 0 (or "false") for the remaining area. The binary matrix multiplied by the density matrix can be used to analyze error only in the liquid-flow area or to compare the free-surface levels.

\section{EXPERIMENTAL WORK}

Benchmarking laboratory tests of double-dam-break type were conducted to evaluate the validity of twodimensional multiphase numerical simulations with the proposed methodology. Fig. 2 and Fig. 3 illustrate the experimental setup: an acrylic container with vertical gates and a light diffuser in the back wall, a set of LED lights that uniformly illuminate the acrylic container from the back wall, and a highspeed camera arranged on a tripod and linked to PC software for its operation. To contrast the two liquid 
phases, the saltwater is colored with organic vegetable dye so that changes in density are negligible (Fig. 2).

The acrylic container is $1.1 \mathrm{~m}$ in length, $0.40 \mathrm{~m}$ in height, and $0.10 \mathrm{~m}$ in width. Two vertical rigid slides (Gate 1 and Gate 2) divide the container into three compartments of the same volume (left CL, central $\mathrm{CC}$, and right $\mathrm{CR}$ ) that are opened from the top, i.e., free-surface condition (Fig. 2). Prior to the beginning of the tests, $\mathrm{CL}$ and $\mathrm{CR}$ were partially filled with freshwater $\left(\rho_{w} \approx 1000 \mathrm{~kg} / \mathrm{m}^{3}\right)$ to a depth of $0.20 \mathrm{~m}$, whereas $\mathrm{CC}$ was filled with saltwater $\left(\rho_{s w} \approx\right.$ $1030 \mathrm{~kg} / \mathrm{m}^{3}$ ) to a depth of $0.10 \mathrm{~m}$, as illustrated in Fig. 3. The water densities were measured with a $151 \mathrm{H}$ hydrometer of $0.001 \mathrm{~kg} / \mathrm{m}^{3}$ resolution.

To record the fluid motion during the liquid phase mixing process, a high-speed camera (model HiSpec1 linked to the software-based ImageBLITZ® Auto Trigger) was installed $\sim 2.5 \mathrm{~m}$ from the container, with its center aligned to the tank center and covering a horizontal field of view (FOVx) of $\sim 1.4 \mathrm{~m}$ (Fig. 3). Grayscale images were acquired at a frame rate of 500 fps (frames per second) over $12 \mathrm{~s}$.

Calibration image acquisition procedures are subject to error caused by lens and perspective distortions. For image-based systems, a typical measure of accuracy is the reprojection error (Semeniuta, 2016), which can be expressed by the root mean square error (RMSE) of the projection, which is computed between real and projected pixel coordinates. In this study, the camera calibration was based on the approach proposed by Zhang (2000), and the accuracy in the acquisition was defined by a projection error in the range of $0.1328 \mathrm{~mm}$ to 0.1748 $\mathrm{mm}$, with a deviation (determined by the RMSE) of $0.1404 \mathrm{~mm}$.

The illumination at the back of the container was carefully set with three LED lamps equally spaced to enhance the contrast between fluid phases and to minimize shadows that could affect image processing. To allow a better distribution of the light, a sheet of paper was used to cover the back face of the container (see the illumination configuration in Fig. 2, with front and top-view shown in Fig. 3).

The tests consider the sudden and synchronized release at $t=0.0 \mathrm{~s}$ of Gates 1 and 2 until they attained a maximum height of $0.05 \mathrm{~m}$ (see aperture distance in Fig. 3). This allows the fresh water in CL and $\mathrm{CR}$ to interact with the saltwater in $\mathrm{CC}$ due to the different water levels of each compartment (i.e., by the action of gravity). The simultaneous release of the two gates attained an average opening time of $\sim 50 \mathrm{~ms}$.

Several repetitions of the study case were performed. Only experiments with simultaneous gate opening, providing symmetrical flow behavior, were selected. Tests with non-symmetrical flow or exceeding $60 \mathrm{~ms}$ of gate aperture time were disregarded. Hence, eighteen tests were considered for validation purposes.

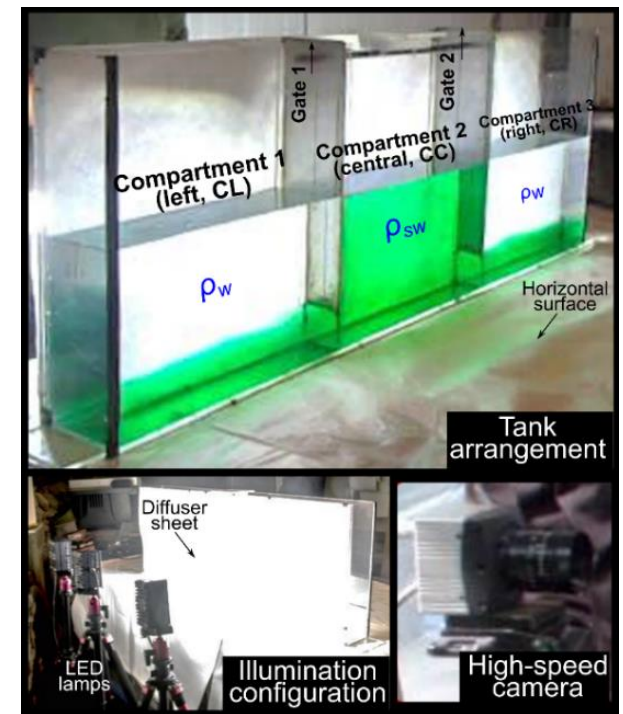

Fig. 2. Experimental setup. (above). Illumination configuration (below-left). High-speed camera used in the experiments (below-right).

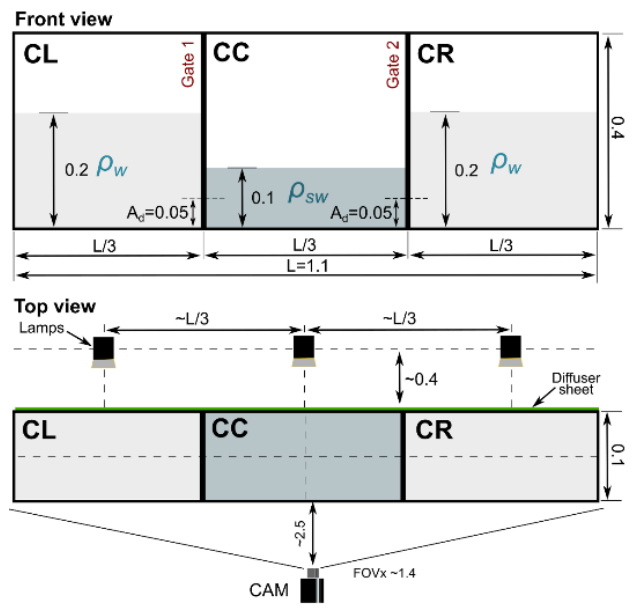

Fig. 3 Dimensions of the experimental installation, camera and lamps positions. Front view (above). Top view (below).

\section{NUMERICAL APPROACH}

The mathematical models used in this research are included in OpenFOAM ${ }^{\circledR}$ software, a free, opensource CFD toolbox, which has gained popularity due to its multiple advantages over free and proprietary CFD codes. This software solves the Navier-Stokes Equations (NSE) in a discrete manner, supported by the Finite Volume Method (FVM), which works by integrating the partial differential equations to be solved. The surface integrals of normal fluxes in FVM guarantee the conservation properties through the domain, allowing the use of complex geometries and unstructured meshes without a coordinate transformation.

This section describes the mathematical model and the governing equations of the numerical simulations. Furthermore, it presents a detailed 
description of the numerical setup of the study case, considering three different turbulence models.

\subsection{Governing Equations}

The Navier-Stokes Equations (NSE) are non-linear equations that accurately describe the fluid-flow motion through momentum and continuity equations (Ferziger and Peric, 2002). The numerical solution of the NSE is a demanding computer task, so simplifications are usually made. An approximated solution in CFD modelling is given by the ReynoldsAveraging process. The resulting averaged equations are known as the Reynolds Averaged Navier-Stokes (RANS) equations. If the flow variable is the velocity, $U$, it is separated into the mean component $\bar{U}$ and the fluctuating component $\mathrm{U}^{\prime}$, i.e., $\mathrm{U}(\mathbf{x}, \mathrm{t})=$ $\bar{U}(x)+U^{\prime}(x, t)$, where $x=(x, y, z)$ is the position of the vector. This is done analogously for the density, $\mathrm{p}^{*}(\mathbf{x}, \mathrm{t})=\overline{\mathrm{p}}(\mathbf{x})+\mathrm{p}^{\prime}(\mathbf{x}, \mathrm{t})$. The decomposed terms are then introduced in the momentum equation for incompressible flows. The average of this equation is done considering that the mean of the fluctuating component is equal to zero $\left(\overline{\mathrm{U}}^{\prime}=0\right)$. Therefore, the RANS equations can be written as in Eq. (2) and Eq. (3) for coordinate-free form and incompressible flows:

$$
\nabla \cdot \mathrm{U}=0
$$

$\frac{\partial \rho \mathrm{U}}{\partial \mathrm{t}}+\nabla \cdot(\rho \mathrm{UU})=\nabla \cdot(\mu \nabla \mathrm{U}-\rho \tau)-\nabla(\mathrm{p})+\rho \mathrm{g}+\sigma \kappa_{\alpha} \nabla \alpha$

where $U$ is the flow velocity, $\rho$ the density, $\mu$ the dynamic viscosity, $p$ is the pressure, $g$ the acceleration due to gravity, and $\tau$ is the specific Reynolds stress tensor defined by the Boussinesq Approximation (Eq. (4)):

$\tau=\frac{2}{\rho} \mu_{\mathrm{t}} \mathrm{S}-\frac{2}{3} \mathrm{kI}$

where $\mu_{\mathrm{t}}$ is the dynamic eddy viscosity, $\mathrm{S}=\frac{1}{2}(\nabla \mathrm{U}+$ $\left.(\nabla \mathrm{U})^{\mathrm{T}}\right)$ is the strain rate tensor, $\mathrm{k}$ the turbulent kinetic energy per unit mass, and I the Kronecker delta. Equation (4) relates the Reynolds stresses to the closure of the RANS equations by considering a turbulence model.

The expression $(\mu \nabla U-\rho \tau)$ in Eq. (3) corresponds to the total shear stress. The last term in Eq. (3) represents the surface tension effect, where $\sigma$ is the surface tension coefficient, $\kappa_{\alpha}$ is the surface curvature, and $\alpha$ represents a scalar field for the identification of the different phases. The value of $\alpha$ is obtained through the VOF (Volume Of Fluid) method detailed by (Berberović et al. 2009). This approach tracks each fluid through every cell in a computational grid using a scalar function that takes the value of zero when the cell is filled by one fluid phase, and one for the other fluid phase. Values between zero and one are used to define the fluid interface. For the free-surface fluid system to be considered, $\alpha=0$ stands for the air phase, whereas $\alpha=1$ represents the liquid phase. The intermediate values represent the fluids interface (Fig. 4). The distribution of $\alpha$ is modelled with the convective equation described in Eq. (5): $\frac{\partial \alpha}{\partial \mathrm{t}}+\nabla \cdot(\alpha \mathrm{U})+\nabla \cdot\left(\mathrm{U}_{\mathrm{r}} \alpha(1-\alpha)\right)=0$

where $U_{r}$ is a relative velocity described as $U_{r}=$ $\mathrm{U}_{\mathrm{l}}-\mathrm{U}_{\mathrm{g}}$, where $\mathrm{l}$ and $\mathrm{g}$ denote the liquid and gas phases for a free-surface fluid system, respectively. The last term on the left side of Eq. (5) belongs to a compression term that limits the interface width.

\begin{tabular}{|c|c|c|c|c|}
\hline 0 & 0 & 0 & 0 & 0 \\
\hline 0.8 & 0.8 & 0.5 & 0.02 & 0 \\
\hline 1 & 1 & 1 & 0.5 & 0 \\
\hline 1 & 1 & 1 & 0 & 0 \\
\hline \multicolumn{4}{|c|}{ Possible interface } \\
locations
\end{tabular}

Fig. 4. Representation of the VOF method for tracking the fluid phases.

The numerical modelling of a three-phase fluid system, which consists of two liquid miscible phases and one gaseous phase, works by solving the liquid phases and their mixing separately, after which they are incorporated as a single fluid phase in the VOF method. For this reason, the model introduces a supporting equation for the conservation of scalar quantities. This equation is written in terms of a scalar $\varphi$ as shown in Eq. (6):

$\frac{\partial \rho \phi}{\partial \mathrm{t}}+\nabla \cdot(\rho \phi \mathrm{U})=\nabla \cdot(\mathrm{D} \nabla \phi)+\mathrm{q}_{\phi}$

where $D$ is the diffusivity of the quantity $\phi$ and $q_{\phi}$ is a source/sink term of $\phi$. The first term on the left side of Eq. (6) is known as the temporal term and the second one as the convective term. On the right side of the equation, the first term belongs to the diffusive term and the second is any source/sink term. This equation allows the representation of scalar quantities in the model, such as the different fluid phases involved. The diffusivity term, $D$, is important in defining the mixing between the liquid phases.

Since NSE equations are a non-closed set of equations, turbulence models must be introduced. The numerical results in a CFD simulation may vary significantly depending on the turbulence model selected. Therefore, evaluating the most suitable turbulence model is important in the validation process. RANS and Large Eddy Simulation (LES) models are two of the most common turbulence models used in industry and engineering already incorporated within the OpenFOAM ${ }^{\circledR}$ framework.

Three turbulence models were tested in this work: i) zero-equation RANS model, ii) two-equation (kepsilon) RANS model, and iii) LES model. The zeroequation model sets the turbulent viscosity as a function of the velocity field, whereas two-equation models add two additional transport equations to represent the turbulent properties of the flow. One of the transport variables is the turbulent kinetic energy, which is represented by $\mathrm{k}$. The second variable 


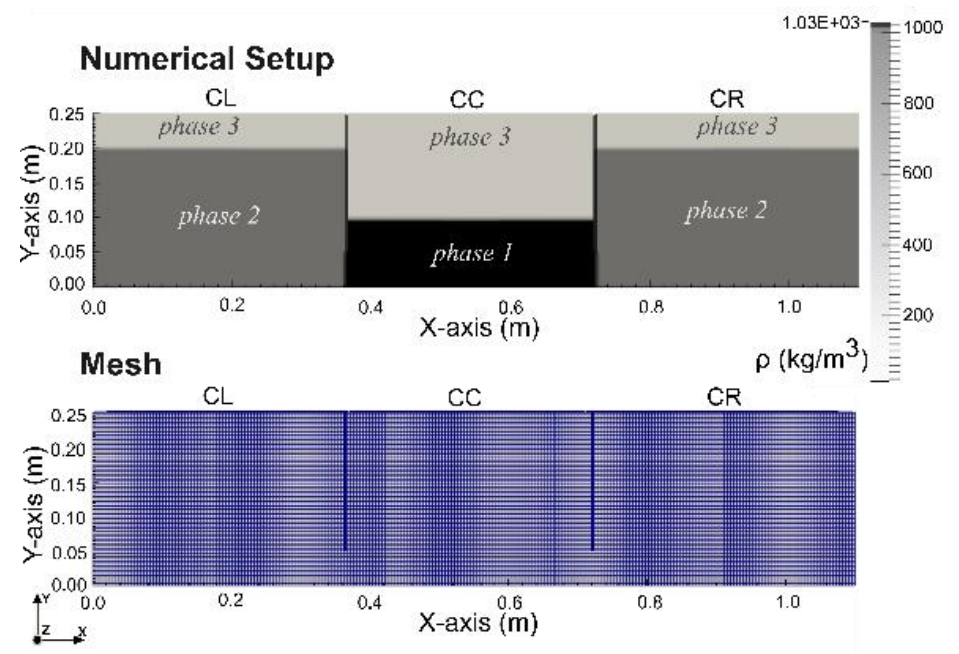

Fig. 5. Numerical setup (above) and mesh grid employed in the numerical simulations (below) of the benchmarking experimental study case.

depends on the model type being used (e.g., k- $\varepsilon$, where $\varepsilon$ represents the turbulent dissipation and determines the rate of dissipation of the turbulent kinetic energy).

The LES turbulence approach solves the largest scale motions of the flow and models the small-scale motions. Although LES was not feasible for practical engineering problems in the $70 \mathrm{~s}$ and $80 \mathrm{~s}$ (Mcdonough, 2007), the transition from RANS to LES was made easier thanks to the development of High-Performance Computing technology and developments in parallel-processor computers (Kobayashi and Tsubokura, 2011), so that this is now one of the most promising turbulence simulations for engineering purposes.

\subsection{Numerical Setup}

The laboratory case was numerically modeled with interMixingFoam, which is an OpenFOAM ${ }^{\circledR}$ native solver. InterMixingFoam solver allows the handling of multiphase fluid-flow, i.e., three fluid phases, two of which are liquid and miscible (e.g., two water phases of different density), and a third phase which is gaseous and incompressible. In this solver, the liquid-phase mixing is obtained through the conservation of scalar quantities (Eq. (6)). For its part, the interface between liquids and the gaseous phase is calculated with Eq. (5). These equations are coupled with the NSE equations to describe the fluidflow motion.

The domain of the numerical setup was configured similarly to that of the laboratory tests by assuming an installation of $1.10 \mathrm{~m}$ in length, $0.25 \mathrm{~m}$ in height, and $0.10 \mathrm{~m}$ in width (Fig. 5). As described in the experiments, for the initial conditions, the central compartment (CC, phase 1) was filled with a fluid with $\rho_{s w} \approx 1030 \mathrm{~kg} / \mathrm{m}^{3}$ at $0.10 \mathrm{~m}$ depth. The two remaining compartments (CL, CR, phase 2) were filled with a fluid with $\rho_{w} \approx 1000 \mathrm{~kg} / \mathrm{m}^{3}$ at $0.20 \mathrm{~m}$ depth. The rest of the computational domain was filled by a third phase, i.e., air with $\rho_{a} \approx 1 \mathrm{~kg} / \mathrm{m}^{3}$
(Fig. 5). The velocity field for all phases was initially set to $0 \mathrm{~m} / \mathrm{s}$ and the gravitational acceleration was set to $-9.81 \mathrm{~m} / \mathrm{s}^{2}$ and acted in the vertical direction

Transport properties, such as kinematic viscosity $(v)$, density $(\rho)$, and surface tension $(\sigma)$ were defined as constant for each fluid phase. The molecular diffusivity $(D)$ is a temperature-dependent value; however, it was considered constant since no temperature variations are taken into account. The value of $D$ was taken from Holz et al. (2000) for a temperature of $15^{\circ} \mathrm{C}$, resembling the temperature in most of the laboratory tests. Surface tension and kinematic viscosity values were taken from Vinš et al. (2019) for a temperature of $15{ }^{\circ} \mathrm{C}$. Table 1 summarizes the input data for the case setup.

The opening of the gates between compartments was considered instantaneous. The interaction of the fluids in the three water compartments occurred for $t$ $>0 \mathrm{~s}$. During the development of the laboratory tests, it was verified that the most significant fluid motion and phase mixing occurred in the first $5 \mathrm{~s}$; thus, such a time-lapse was considered for validation purposes.

Table 1 Transport properties defined for the fluid phases and molecular diffusion coefficient for the numerical model setup (values obtained experimentally and from Holz et al. 2000, and Vinš et al. 2019)

\begin{tabular}{|c|c|c|c|}
\hline Phase property & Freshwater & Saltwater & Air \\
\hline Density $\left(\mathrm{kg} / \mathrm{m}^{3}\right)$ & 1000 & 1030 & 1 \\
\hline $\begin{array}{c}\text { Kinematic } \\
\text { viscosity }\left(\mathrm{m}^{2} / \mathrm{s}\right)\end{array}$ & $1.007 \mathrm{E}-6$ & $1.082 \mathrm{E}-6$ & $\begin{array}{c}1.48 \mathrm{E}- \\
5\end{array}$ \\
\hline $\begin{array}{c}\text { Surface tension } \\
(\mathrm{N} / \mathrm{m})\end{array}$ & 0.07349 & 0.07416 & - \\
\hline $\begin{array}{c}\text { Molecular } \\
\text { diffusion } \\
\text { coefficient } \\
\left(\mathrm{m}^{2} / \mathrm{s}\right)\end{array}$ & $1.26 \mathrm{E}-9$ & - \\
\hline
\end{tabular}


No-slip boundary condition was considered for the walls of the container, i.e., the fluid have zero velocity at the solid boundaries. The top boundary allows inflow and outflow, which means a freesurface condition.

\subsubsection{Mesh Independence Study}

The mesh resolution was selected considering a mesh independence study based on the comparison of physical parameters (i.e., speed and pressure in this study) for different mesh resolutions and turbulence models.

Three mesh configurations were tested: Mesh A (MA), Mesh B (MB), and Mesh C (MC). MA corresponds to the finest mesh, MC to the coarsest, and $\mathrm{MB}$ to an intermediate mesh resolution. The refinement factor was set to $\sqrt{2}$ for spatial and temporal discretization, since both discretization schemes are of second-order (Stern et al. 2001). The mesh was designed to keep an aspect ratio close to one. Table 2 lists the characteristics of the three meshes.

Table 2 Mesh characteristics for the mesh independence study

\begin{tabular}{|c|c|c|c|c|}
\hline Mesh & Direction & $\begin{array}{c}\text { Dimension } \\
(\mathrm{m})\end{array}$ & $\begin{array}{c}\text { Cell } \\
\text { number }\end{array}$ & $\begin{array}{c}\text { Cell size } \\
(\mathrm{cm})\end{array}$ \\
\hline \multirow{4}{*}{ MA } & $\mathrm{X}$ & 1.1 & 310 & 0.35 \\
\cline { 2 - 5 } & $\mathrm{Y}$ & 0.25 & 70 & 0.36 \\
\cline { 2 - 5 } & $\mathrm{Z}$ & 0.1 & 28 & 0.36 \\
\hline \multirow{4}{*}{ MB } & $\mathrm{X}$ & 1.1 & 220 & 0.50 \\
\cline { 2 - 5 } & $\mathrm{Y}$ & 0.25 & 50 & 0.50 \\
\cline { 2 - 5 } & $\mathrm{Z}$ & 0.1 & 20 & 0.50 \\
\hline \multirow{3}{*}{ MC } & $\mathrm{X}$ & 1.1 & 156 & 0.71 \\
\cline { 2 - 5 } & $\mathrm{Y}$ & 0.25 & 36 & 0.69 \\
\cline { 2 - 5 } & $\mathrm{Z}$ & 0.1 & 14 & 0.71 \\
\hline
\end{tabular}

The velocity module (speed) and the pressure were obtained at one specific point within the domain, compared for the three grids, and for each of the three turbulence models. Since the area within the gate aperture has significant velocity and pressure gradients, the coordinate $(0.366,0.001,0.05)$ was selected as the probe point. Fig. 6 shows the comparison of the speed through time for each turbulence model at the probe point.

For the speed series, the Mean-Absolute errors (MAE), in $\mathrm{m} / \mathrm{s}$, were calculated as $\frac{1}{n} \sum_{i=1}^{n}\left|e_{i}\right|$, where $\left|e_{i}\right|$ is the absolute error at $t=i$, and $\mathrm{n}$ is the number of data. The finest mesh (MA) was considered the reference mesh. MAE were also calculated for the pressure field, in $\mathrm{Pa}$ (Table 3).

Table 3 MAE of each turbulence model

\begin{tabular}{|c|c|c|c|c|}
\hline & \multicolumn{2}{|c|}{ Speed (m/s) } & \multicolumn{2}{c|}{ Pressure (Pa) } \\
\hline & $\mathrm{MB}$ & $\mathrm{MC}$ & $\mathrm{MB}$ & $\mathrm{MC}$ \\
\hline $\begin{array}{c}\text { Zero- } \\
\text { equation }\end{array}$ & 0.011 & 0.039 & 10.11 & 16.00 \\
\hline $\begin{array}{c}\text { Two- } \\
\text { equation } \\
\text { (k- } \varepsilon \text { ) }\end{array}$ & 0.018 & 0.041 & 5.89 & 14.24 \\
\hline LES & 0.010 & 0.040 & 10.34 & 17.01 \\
\hline
\end{tabular}

Fig. 7 shows the calculated yPlus (dimensionless wall distance) values for the three turbulence models tested when using MB. In the zero-equation model, yPlus ranges between 0 and 3.27; for the twoequation (k-epsilon) model between 29.62 and 272.93, and for the LES model between 0 and 53.22.

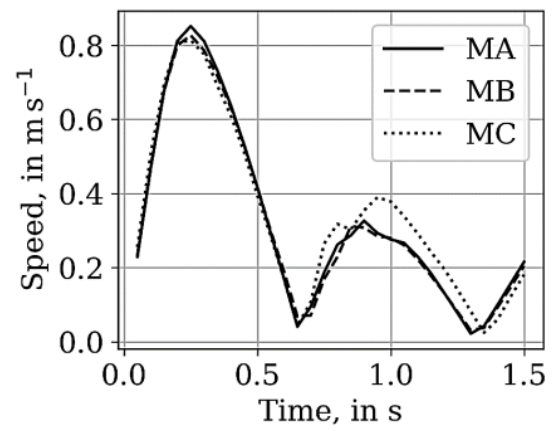

a)

Zero-equation

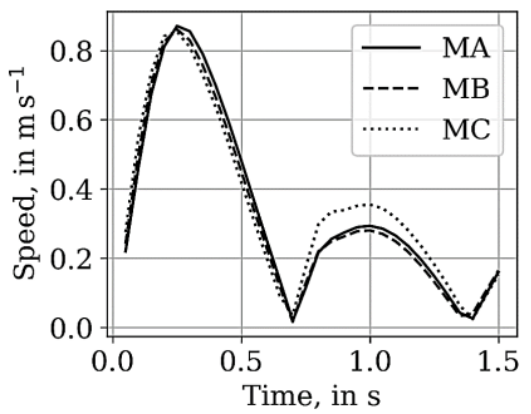

b) Two-equation (k-epsilon)

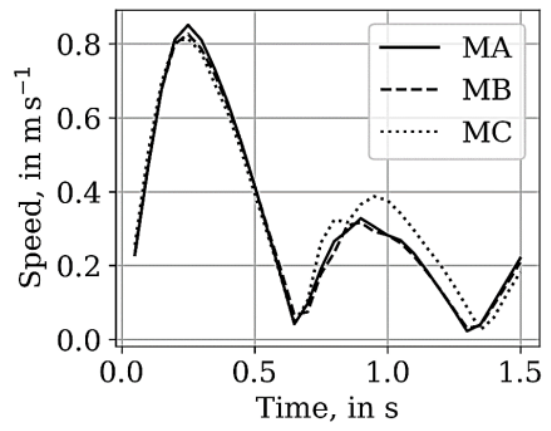

c) LES

Fig. 6. Speed in the three turbulence models and comparison for the three mesh configurations.

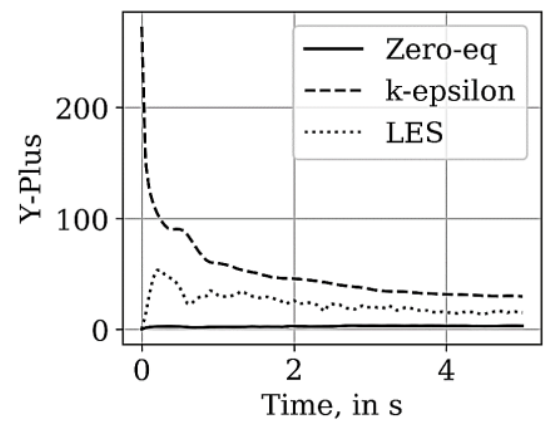

Fig. 7. Average yPlus values through time for Mesh B (MB). 


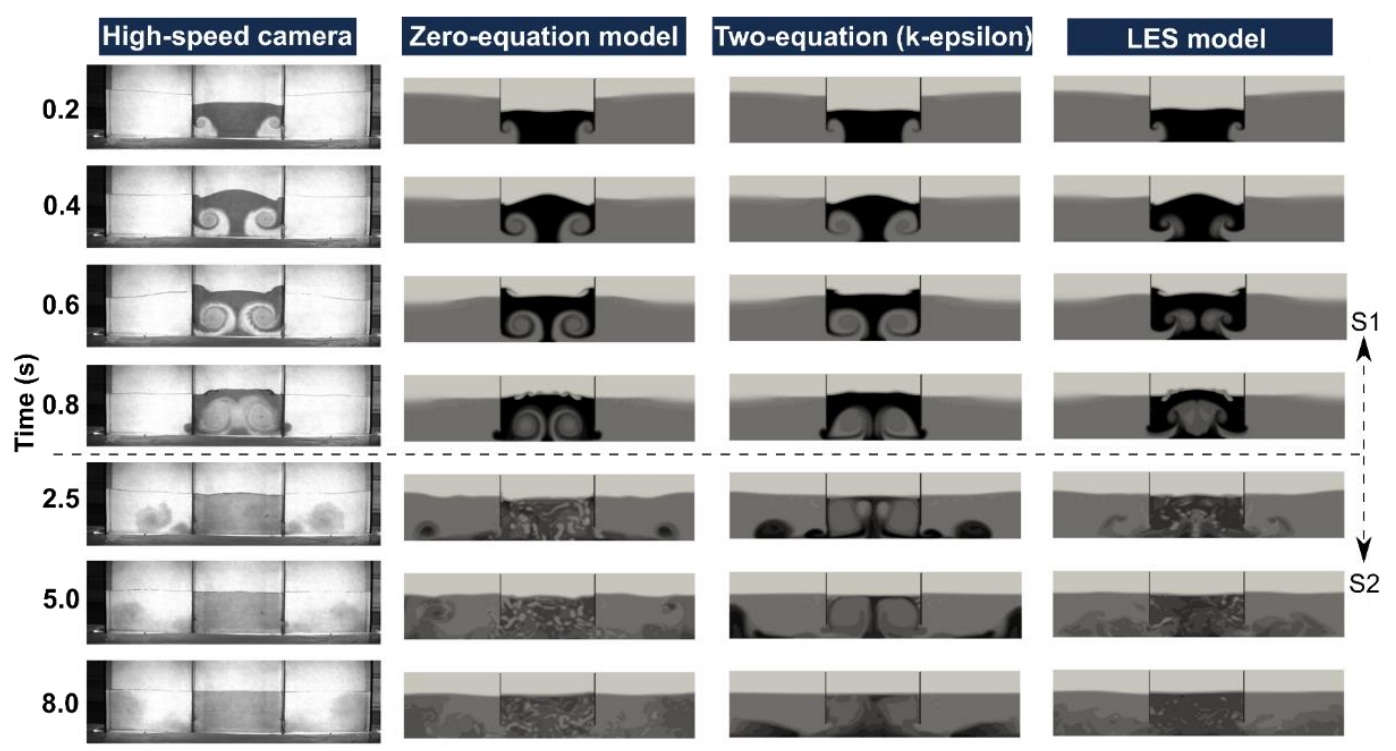

Fig. 8. Comparisons of snapshots at different time instants for the experimental results (Column 1), and the numerical results obtained with the three turbulence models (Columns 2-4). S1: $0.0<t<0.8$ s. S2: $t$ $>0.8 \mathrm{~s}$.

MA requires a significant running time when compared to $\mathrm{MB}$ and $\mathrm{MC}$, and the results are very similar to those of MB. Therefore, MB was selected to perform the validation process with each turbulence model.

\section{RESULTS AND DISCUSSION}

\subsection{Qualitative Comparison}

Images from the experiment were compared against post-processing images of the numerical simulation. Since different processes were observed to be dominant in terms of turbulence development, the double-dam-break problem was divided into two sections: the incipient mixing $(0.0<\mathrm{t}<0.8 \mathrm{~s}), \mathrm{S} 1$, and the full blend $(t>0.8 \mathrm{~s}), \mathrm{S} 2$. Fig. 8 shows the high-speed camera images referring to $\mathrm{S} 1$ and $\mathrm{S} 2$, and the comparison against the numerical simulations with different turbulence models.

$\mathrm{S} 1$ is dominated by the pressure gradient between the sections of the tank (i.e., different hydraulic head between the compartments), and features the development of large eddies and strong free-surface fluctuations. During this process, the free-surface develops a bell-shaped curve. Eddies caused by the entrance of freshwater from CL and CR into the CC are of up to $\sim 15 \mathrm{~cm}$ diameter, occupying most of the $\mathrm{CC}$ area.

On the other hand, mixing between the phases and progressive stabilization of the fluid-flow take place during S2. The larger eddies observed in S1 convert into increasingly smaller eddies in the central compartment of the tank. In the lateral compartments, medium-sized eddies of about $\sim 10 \mathrm{~cm}$ in diameter form due to the entry of saltwater, and subsequently decrease as the mixing between phases takes place.

The results of the Zero-equation RANS turbulence model show, tentatively, the closest representation of the laboratory conditions. For S1, both the freesurface and eddies in the simulation evolve similarly to the experimental results. Mixing between the liquid phases in S2 is also close to that shown by the numerical model. However, a slight underestimation of the energy dissipation due to turbulence can be inferred from the stabilization lapse of the fluid-flow. The mixing between phases in S2 is faster and more chaotic if compared to the two-equation and LES models, but with a more satisfactory development of the irregular interface between salt- and freshwater.

On the other hand, the results of the two-equation (kepsilon) model seem to be, from a visual and qualitative perspective, less accurate than the other models. The energy lost due to turbulence seems to be overestimated. Moreover, there is less mixing between the liquids when compared against the chaotic behavior observed in the experimental results and in the other turbulence approaches.

For its part, the LES turbulence model provided significantly different results from those observed in the laboratory tests, particularly for S1. The largescale eddies do not evolve completely and quickly vanish in the central section of the container. However, in S2 the phase-mixing resulted more homogeneous and quite similar to the experimental results. The mixing is less disordered than the zeroequation simulation but it is not as passive as in the two-equation simulation. The stabilization of the fluid motion seems to be consistent with the observations in the laboratory tests.

From the three turbulence models analyzed, a qualitative inspection suggests that the Zero- 
equation turbulence model best represents the physics of the experiments.

\subsection{Quantitative \\ Comparisons: Application of the Image-Based Multiphase CFD Validation Methodology}

The eight steps of the proposed image-based validation methodology presented in Fig. 1 were followed to obtain the error of the numerical simulations. The steps of the methodology were addressed as follows:

i. Acquisition of graphical data: The first step of the methodology demands the acquisition of experimental and numerical image-based data. These data were obtained through the procedures described in Sections 3 and 4.

ii. Image edition: The images captured by the camera were cropped, providing focus on the acrylic container and the fluid motion inside (Fig. 9a). Images recorded after the analysis time (i.e., $5 \mathrm{~s}$ ) were deleted, as well as those before the gates were completely opened. Post-processing images were also cropped to match the study area of the experiment images.

iii. Frequency matching: To match the time steps between the experiment recording (500 fps) and the numerical results (20 fps), subsampling was required. Thus, images of the experiment were considered every 25 frames to match the timestep of the post-processing images.

iv. Pixel matching: To obtain the same amount of pixels between images, an interpolation was performed. The pixel matrix resolution of the post-processing snapshots was interpolated with the pixel resolution of the experiment images.

v. Calibration in time-zero: Each of the eighteen experiments and the three numerical simulations were calibrated according to the time-zero frame, i.e., before the opening of the gates. For this purpose, a pixel within the central section of the container was given a density value of $\rho_{1}=$ $1000 \mathrm{~kg} / \mathrm{m}^{3}$, while a pixel of a section with saltwater was given a density of $\rho_{2}=1030 \mathrm{~kg} /$ $\mathrm{m}^{3}$. As observed in Fig. 9b, the darkest tone in CC corresponds to $\rho_{1}$ while in LC or RC the lighter tone corresponds to $\rho_{2}$.

vi. Generation of density matrices: The color scale was calibrated based on a linear interpolation between $\rho_{1}$ and $\rho_{2}$. A density value, according to the calibration, was given to each pixel in the high-speed camera images and the postprocessing images.

vii. Averaging of the experimental density matrices (optional): The density matrices of the eighteen experiments performed (as repetitions of the study case) were averaged for each time step, yielding a single-averaged set of density matrices. This averaged experimental result is compared against each of the three turbulence approaches of the numerical simulations. viii. Generation of error matrices: The averaged density matrix from the laboratory tests $\left(\overline{\rho_{\text {exp }}}\right)$ was compared against the density matrix from the numerical simulation $\left(\rho_{\text {sim }}\right)$. The relative error for each time step was obtained by considering the absolute value of the difference of $\overline{\rho_{\exp }}$ and $\rho_{\text {sim }}$ and then dividing this difference by the density gradient (i.e., $\Delta \rho=30 \mathrm{~kg} / \mathrm{m}^{3}$ ), as shown in Eq. (1).

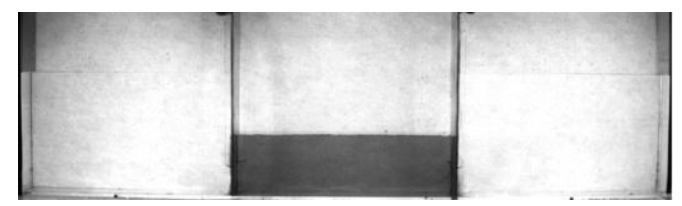

(a)

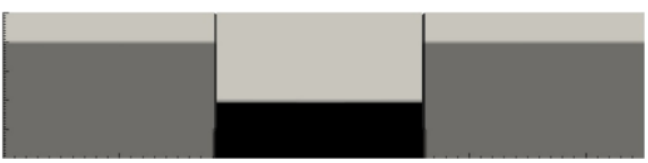

(b)

Fig. 9. Cropped images of the experimental and numerical results. (a) Example of an image captured by the high-speed camera at $t=0 \mathrm{~s}$. (b)

Example of an image of the density field obtained in the post-processing of the numerical simulation at $\mathrm{t}=0 \mathrm{~s}$.

After the generation of the error matrices, the relative error through time was plotted to observe its evolution and to compare the results obtained with the different turbulence models. Fig. 10 shows the error matrices obtained for each turbulence model for different time instants $(0.3,0.6,1.0,1.5,2.5$, and 5.0 $\mathrm{s})$. In the plots, each pixel color represents the relative error estimated employing Eq. (1).

In general, the error increases gradually with time, particularly in those regions where eddies develop and the mixing occurs. The darkest color is considered as an indicator of better agreement of the numerical results in representing the physics of the experiments. Even though the error was greater for longer times, the averaged relative error in the mixing area is lower than $40 \%$, approximately.

Time zero has a theoretical error of $0 \%$; however, there are some areas where the error is slightly higher. These high values might be attributed to the noise introduced in the experimental data due to the illumination setup and the sampling rate considered in the present work. These are important considerations for 2D hydrodynamic experiments using high-speed video to analyze the flow evolution (Hernández et al. 2018). Hernández et al. (2018) suggested that the natural reflectance of the fluid has significant importance since it may cause irregular intensity values in different areas of the images. Moreover, high-speed sampling rates could increase the signal-to-noise ratio (SNR), particularly when the illumination is insufficient. These factors might have introduced some variations in the error calculation. 


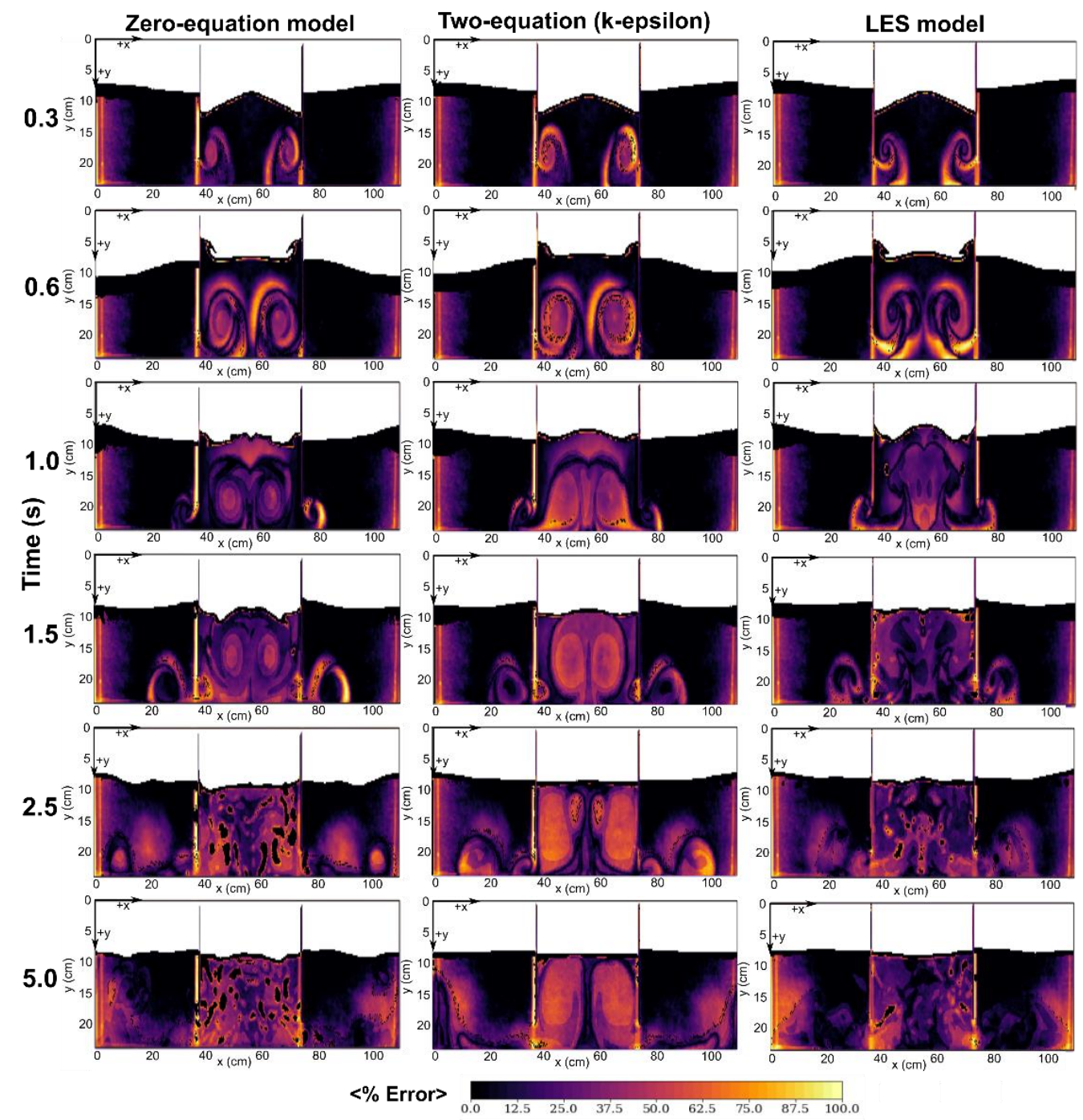

Fig. 10. Error matrices for different time steps between experiments, and the three turbulence numerical models tested.

To analyze the variation of the relative error through time for the three turbulence models, only the interaction between the two liquid-miscible phases was considered. The area occupied by the liquid phases and that occupied by air was first identified. This step is straightforward when using the postprocessing images because the gray-scale tonalities clearly differentiate the liquid phases and the gaseous phase (see Fig. 9b). Thus, the post-processing images were used to generate a binary matrix, where 1 was given to the liquid phases and 0 to the gaseous one. The multiplication of the binary matrix with the density matrices of both experiment and numerical images provided data for the liquid flow interactions only. The zero values were then deleted (replaced by not-a-value) to obtain the relative errors in the areas of interest.

The domain was divided into the left, central and right compartments (i.e., $\mathrm{LC}, \mathrm{CC}$ and $\mathrm{RC}$ ) for which the averaged relative error per section was estimated.
Thereby, the time series of averaged relative error for each compartment can be visualized, as shown in Fig. 11a, b and c, respectively.

For the three turbulence models (Fig. 11a-c), the error within the central compartment (CC) tends to increase with time in the first 3.0 seconds and later tends to stabilize. In general, averaged relative error oscillates between $\sim 2$ to $\sim 18 \%$ in the lateral compartments (RC and LC) and between $\sim 2$ to $\sim 39$ $\%$ in the central compartment (CC). The gap between RC and LC errors $(<6 \%)$ may be due to the symmetry of the experiment.

Regarding the minimum and maximum errors for the three turbulence models, it can be noted that for the zero-equation model, averaged relative error of $\sim 2 \%$ and $\sim 19 \%$ were obtained for the RC and LC, and of $\sim 2 \%$ and $\sim 32 \%$ for the central compartment CC. For S1 $(0.0<\mathrm{t}<0.8 \mathrm{~s})$ and $\mathrm{S} 2(\mathrm{t}>0.8 \mathrm{~s})$, the CC presented mean averaged errors of $\sim 15 \%$ and $\sim 28 \%$, respectively. Averaging the three 
compartments, a global averaged relative error of $\sim 17 \%$ was obtained. Even though the zero-equation turbulence model was considered to perform best in the qualitative inspection, it produced the second highest averaged relative error in the present quantitative results.

On the other hand, for the two-equation (k-epsilon) turbulence model, the minimum and maximum averaged relative errors were of $\sim 2 \%$ and $\sim 18 \%$ for the lateral compartments, and of $\sim 3 \%$ and $\sim 39 \%$ for the CC. Averaging the three compartments, a global error of $\sim 19 \%$ was obtained. For S1 and S2, the central compartment provided averaged relative errors of $\sim 18 \%$ and $\sim 36$ $\%$, respectively.

Finally, for the LES turbulence model, minimum and maximum averaged relative errors of $\sim 2 \%$ and $\sim 17 \%$ for the lateral compartments, and $\sim 2 \%$ and $\sim 26 \%$ central compartments were obtained, respectively. A global error of $\sim 14 \%$ was found from the averaging of the three compartments. For S1 and $\mathrm{S} 2$ of the experiment, the CC presented averaged relative errors of $\sim 16 \%$ and $\sim 20 \%$ respectively.

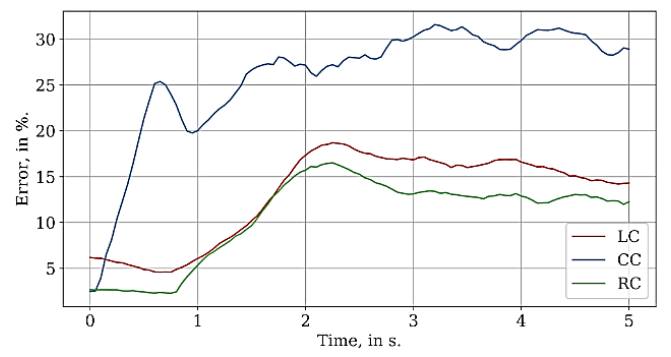

a) Zero-equation

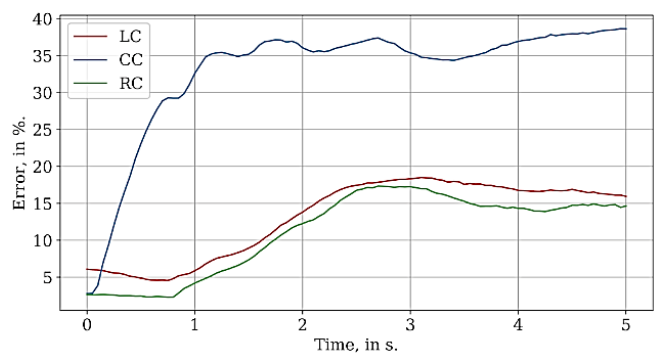

b) Two-equation (k-epsilon)

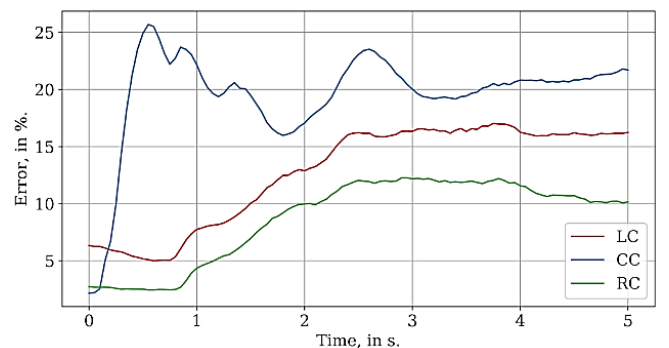

c) LES

Fig. 11. Time series of averaged relative error (in $\%$ ) for the three turbulence models. High-speed camera photographs vs. images of the postprocessing.

\section{DisCuSSION AND RECOMMEND- ATIONS}

In contrast to the qualitative analysis, the quantitative results suggest that the LES model is the most accurate in representing the mixing process through time, in terms of averaged relative error. It is worth mentioning that the highest error of the LES model occurred in S1, where the large eddies are generated, unlike S2, where eddies had already developed. Moreover, the error exhibits a more stable performance throughout the modelling, if compared to the RANS models.

The results show that the methodology presented here may yield different alternatives in the comparison between numerical simulations and experiments; thus, contributing to preliminary validations. Among these alternatives, it is possible to perform qualitative analyses by visually comparing different stages of the phenomena, and quantitative analyses by estimating the averaged relative errors between image-based results from the simulations and experiments. In the latter, it is possible to analyze the global averaged relative errors either at specific time steps (i.e., from snapshots at the same time instant) or along the time series (i.e., from the complete series of snapshots). Moreover, the analysis can be focused on the complete domain, where the fluid interactions occur, or at specific areas or points of this domain.

The methodology could be improved through noise reduction in the acquired image data from experiments, either by improving the illumination setup or enhancing the fluid interfaces differences by coloring both liquid fluids, as suggested by Hernández et al. (2018). With these improvements, the phases could be better differentiated and the error calculation might be reduced.

Moreover, an indirect method to track and validate the free-surface calculation could be conducted if the liquid phases were given different tonalities to air (white background). This would allow a density value to be assigned to each tonality on both experiment photos and post-processing snapshots to assess further the relative error for the complete domain. The free-surface can also be tracked for a specific point by comparing the number of pixels with a higher density (e.g., where $\rho \geq 1000 \mathrm{~kg} / \mathrm{m}^{3}$ ) in the vertical direction of the location of interest.

The methodology can be also extended to similar two-dimensional multiphase flow applications which could also focus, for instance, on the performance of near-wall flow fields but for which further experiments and research might be needed.

\section{Conclusions}

There are different multiphase flow types with differences in pressures, temperatures, densities and flow regimes and types, and therefore there is no specific experimental setup that can validate all types of multiphase flow conditions. The work presented 
here proposes an alternative way of examining the validity of numerical multiphase-flow results, employing image-based methods for the analysis. The main idea is to estimate the relative errors between numerical and experimental image-based results, which are considered as indicators to quantify the degree of validity of the numerical simulations.

The proposed methodology is applicable to liquidliquid-gas interface flows in a two-dimensional (2D) framework, where the fluids have different densities and there is a free-surface condition. It allows analysis of the validity of the numerical data globally (i.e., over the complete domain of fluid interactions), at specific locations (i.e., smaller areas or specific points in the domain), at specific instants of the phenomenon of interest (i.e., comparisons of specific frames), or in the complete time series for the analysis (i.e., by analyzing the complete frames stack).

To demonstrate the application of the proposed methodology, both experimental and numerical research was performed, considering a double-dambreak problem in a rectangular installation (assumed 2D), as the benchmarking study case.

In the experimental phase of the research, three equal-spaced compartments were defined by two vertical dams (gates). Each compartment was filled with fluids of different densities, presenting a freesurface. When the gates were suddenly and simultaneously released, the liquid interfaces interacted, producing mixing. This rapid interaction was captured using high-speed video in systematic and repeatable tests.

In the numerical phase, the benchmarking study case was simulated with OpenFOAM® CFD software. Three turbulence models were considered in the implementation of this methodology: i) the zeroequation RANS model, ii) the two-equation RANS (k-epsilon) model, and iii) the LES model.

Image-based results from the numerical and experimental phases were compared via qualitative and quantitative analyses. The former consisted of comparing various frames of the experiment against the same time-step in the images given by the numerical simulation. The latter consisted of evaluating the averaged relative error between numerical and experimental results, employing the proposed image-based methodology.

The experimental results compared favorably against the numerical results, with global averaged relative errors oscillating between $\sim 17$ and $\sim 19 \%$ for the zero equation and the two-equation (k-epsilon) turbulence schemes, and $\sim 14 \%$ for the LES model. From the results obtained, it can be inferred that the LES model reproduces the mixing between phases more accurately than RANS models; however, zeroequation models seem to be more suitable for capturing the formation of eddies in the initial phase of the experiment. Two equation (k-epsilon) model had the worst behavior in terms of relative errors.
The present methodology can be used as an alternative tool to analyze the degree of validity of numerical multiphase simulations. It is possible to evaluate global averaged relative errors in the complete domain, where the fluid interactions occur, or to focus on particular areas or points inside such a domain, to perform particular and more detailed comparisons. The method can be adapted and extended according to the user's requirements.

\section{ACKNOWLEDGEMENTS}

The first author is grateful for the financial support provided by CONACYT and the EXCEEDSWINDON program during her Master of Science studies. The authors thank the staff of the Grupo de Ingeniería Costas y Puertos of the Instituto de Ingeniería of the II-UNAM for their support in performing the experiments and for their valuable comments.

\section{REFERENCES}

Afrasiabi, M., M. Roethlin and K. Wegener (2018). Thermal simulation in multiphase incompressible flows using coupled meshfree and particle level set methods. Comput. Methods Applied Mechanical Engineering 336, 667-694.

Bai, L., L. Zhou, C. Han, Y. Zhu and W. Shi (2019a). Numerical study of pressure fluctuation and unsteady flow in a centrifugal pump. Processes 7,354 .

Bai, L., L. Zhou, X. Jiang, Q. Pang and D. Ye (2019b). Vibration in a Multistage Centrifugal Pump under Varied Conditions. Shock and Vibration, 1-9.

Bello, O. O., G. Falcone and C. Teodoriu (2007). Experimental validation of multiphase flow models and testing of multiphase flow meters: A critical review of flow loops worldwide. WIT Transactions on Engineering Sciences 56, 97111.

Berberović, E., N. P. Van Hinsberg, S. Jakirlić, I. V. Roisman and C. Tropea (2009). Drop impact onto a liquid layer of finite thickness: Dynamics of the cavity evolution. Physical Review E: Statistical, Nonlinear, and Soft Matter Physics 79, 036306 1-15.

Buliński, P., J. Smolka, S. Golak, R. Przyłucki, M. Palacz, G. Siwiec, B. Melka and L. Blacha (2018). Numerical modelling of multiphase flow and heat transfer within an induction skull melting furnace. International Journal of Heat and Mass Transfer 126, 980-992.

Buradi, A. and A. Mahalingam (2016). Numerical Simulation of Pulsatile Blood Flow in an Idealized Curved Section of a Human Coronary. International Journal of Mechanical and Production Engineering Special Issue 2016, 15-19. 
Cao, Z., J. Xu, D. Sun, J. Xie, F. Xing, Q. Chen and X. Wang (2014). Numerical simulation of modulated heat transfer tube in laminar flow regime. International Journal of Thermal Sciences 75, 171-183.

Chang, C. H., J. S. Lin, C. M. Cheng and Y. S. Hong (2013). Numerical simulations and wind tunnel studies of pollutant dispersion in the urban street canyons with different height arrangements. Journal of Marine Science and Technology 21, 119-126.

Department of Defense (2009). DoD Modeling and Simulation (M\&S) Verification, Validation, and Accreditation (VV\&A), Instruction.

Eça, L. and M. Hoekstra (2008). Code Verification of unsteady flow solvers with method of manufactured solutions. International Journal of Offshore and Polar Engineering 18, 120126.

Eça, L., G. Vaz, A. Koop, F. Pereira and H. Abreu (2016). Validation: What, Why and How, in: Proceedings of the ASME 2016 35th International Conference on Ocean, Offshore and Arctic Engineering OMAE2016 Busan, South Korea.

Ersoy, G., C. Sarica, E. Al-Safran and H. Q. Zhang (2017). Three-phase gas-oil-water flow in undulating pipeline. Journal of Petroleum Science and Engineering 156, 468-483.

Ferziger, J. H. and M. Peric (2002). Computational Methods for Fluid Dynamics, 3rd ed, Vasa. Springer.

Foster, A. S. J., T. Rossetto and W. Allsop (2017). An experimentally validated approach for evaluating tsunami inundation forces on rectangular buildings. Coastal Engineering 128, 44-57.

Greco, M., G. Colicchio and O. M. Faltinsen (2007). Shipping of water on a two-dimensional structure. Part 2. Journal of Fluid Mechanics 581, 371-399.

Greco, M., O. M. Faltinsen and M. Landrini (2005). Shipping of water on a two-dimensional structure. Journal of Fluid Mechanics 525, 309-332.

Hernández-Fontes, J. V., M. A.Vitola, P. de T. T. Esperança and S. H. Sphaier (2018). Assessing shipping water vertical loads on a fixed structure by convolution model and wet dambreak tests. Applied Ocean Research 82, 63-73.

Hernández, I. D., J. V. Hernández-Fontes, M. A. Vitola, M. C. Silva and P. T. T. Esperança (2018). Water elevation measurements using binary image analysis for 2D hydrodynamic experiments. Ocean Engineering 157, 325-338.

Holz, M., S. R. Heil and A. Sacco (2000) Temperature-dependent self-diffusion coefficients of water and six selected molecular liquids for calibration in accurate $1 \mathrm{H}$ NMR PFG measurements. Physical Chemistry Chemical Physics 2, 4740-4742.

ITTC (2008). Uncertainty Analysis in CFD Verification and Validation Methodology and Procedures, 25th ITTC International Towing Tank Conference 2008.

Jiang, X., A. Lin, A. Malik, X. Chang and Y. Xu (2019). Numerical investigation on aerodynamic characteristics of exhaust passage with consideration of multi-factor components in a supercritical steam turbine. Applied Thermal Engineering 162, 114085.

Kobayashi, T. and M. Tsubokura (2011). Current Status on Large-Eddy Simulation for Engineering Applications 10, 149-152.

Kumar, V. and V. K. D. P. Nigam (2017). Multiphase fluid flow and heat transfer characteristics in microchannels. Chemical Engineering Science 169, 34-66.

Lamas, W. de Q., F. F. Bargos, G. E. O. Giacaglia, F. J. Grandinetti and L. de Moura (2017). Numerical modelling and simulation of multiphase flow through an industrial discharge chute. Applied Thermal Engineering 125, 937950 .

Leporini, M., B. Marchetti, F. Corvaro, G. di Giovine, F. Polonara and A. Terenzi (2018). Sand transport in multiphase flow mixtures in a horizontal pipeline: An experimental investigation. Petroleum 5, 161-170.

Lin, A., Y. Sun, H. Zhang, X. Lin, L. Yang and Q. Zheng (2018). Fluctuating characteristics of airmist mixture flow with conjugate wall-film motion in a compressor of gas turbine. Applied Thermal Engineering 142, 779-792.

Lobovský, L., E. Botia-Vera, F. Castellana, J. MasSoler and A. Souto-Iglesias (2014). Experimental investigation of dynamic pressure loads during dam break. The Journal of Fluids and Structures 48, 407-434.

Mcdonough, J. M. (2007). Introductory Lectures on Turbulence Physics, Mathematics and Modeling, 2nd ed. Departments of Mechanical Engineering and Mathematics University of Kentucky, Kentucky.

Melka, B., M. Gracka, W. Adamczyk, M. Rojczyk, A. Golda, A. J. Nowak, R. A. Białecki and Z. Ostrowski (2018). Multiphase simulation of blood flow within main thoracic arteries of 8year-old child with coarctation of the aorta. Heat and Mass Transfer/Waerme- und Stoffuebertragung 54, 2405-2413.

Moraiti, A. N., Margaris, D. P., N. Panopoulos (2016). CFD Modelling of Air Pollution Motion Inside a Building Block, in: 7th International Conference from "Scientific Computing to Computational Engineering”, Athens, Greece. 
Ostrowski, Z., B. Melka, W. Adamczyk, M. Rojczyk, A. Golda and A. J. Nowak (2016). CFD analysis of multiphase blood flow within aorta and its thoracic branches of patient with coarctation of aorta using multiphase Euler Euler approach. Journal of Physics: Conference Series $745,1-8$

Pessoa, D. R., A. T. J. Finkler, A. V. L. Machado, L. F. L. Luz and D. A. Mitchell (2016). Fluid dynamics simulation of a pilot-scale solid-state fermentation bioreactor. Chemical Engineering Transactions 49, 49-54.

Rodríguez-Ocampo, P. E. (2016). Modelación numérica de flujos multifásicos: interacción del oleaje con descargas submarinas de agua dulce. UNAM, Mexico.

Schmidt, D. and K. Velten (2016). Numerical simulation of bubble flow homogenization in industrial scale wine fermentations. Food and Bioproducts Processing 100, 102-117.

Schmidt, D. and K.Velten (2015). Modeling and simulation of the bubble-induced flow in wine fermentation vessels. BIO Web Conference 5, 02015.

Semeniuta, O. (2016). Analysis of Camera Calibration with Respect to Measurement Accuracy. Procedia CIRP 41, 765-770.

Shi, Y., H. Zhu, J. Zhang, J. Zhang and J. Zhao (2018). Experiment and numerical study of a new generation three-stage multiphase pump Journal of Petroleum Science and Engineering $169,471-484$

Sia, S. F., X. Zhao, Y. Yu and Y. Zhang (2018). Multiphase particle-in-cell simulation in severe internal carotid artery stenosis. Powder Technology 358, 62-67.

Stern, F., R. Wilson and J. Shao (2006). Quantitative $\mathrm{V} \& \mathrm{~V}$ of CFD simulations and certification of CFD codes. The International Journal for Numerical Methods in Fluids 50, 1335-1355.

Stern, F., R. V. Wilson, H. W. Coleman and E. G. Paterson (2001). Comprehensive Approach to Verification and Validation of CFD Simulations-Part 1: Methodology and Procedures. Journal of Fluids Engineering 123, 793-802.

Sun, G., J. Zhang, C. Ma and X. Wang (2016). Startup flow behavior of pipelines transporting waxy crude oil emulsion. Journal of Petroleum Science and Engineering 147, 746-755.

Vinš, V., J. Hykl and J. Hrubý (2019). Surface tension of seawater at low temperatures including supercooled region down to $-25^{\circ} \mathrm{C}$. Marine Chemistry 213, 13-23.

Zhang, Z. (2000). A flexible new technique for camera calibration, IEEE Transactions on Pattern Analysis and Machine Intelligence. 22(11), 1330-1334. 